\title{
Uncommon Common Cold: Intracranial Fungal Ball
}

\author{
Rajiv R Sanji ${ }^{1}$, Reshma S Abey ${ }^{2}$, Aniruddha Tekkatte Jagannatha ${ }^{3}$
}

\begin{abstract}
Aim: To present an unusual clinical opportunity with invasive fungal sinusitis.

Background: Mucormycosis is a rare opportunistic zygomycotic fungal infection occuring mainly in immunocompromised patients. Rhinocerebral mucormycosis is the most common form of the disease.

Case description: Here we present a rare case of intracranial mucormycosis in an immunocompetent patient. The patient presented with left nasal block and recurrent nasal discharge. Nasal endoscopy showed multiple pale polyps. Magnetic resonance imaging findings showed left frontal, ethmoidal, and sphenoid sinusitis with leptomeningeal enhancement.

Management: A dual endonasal-transcranial approach was used by a neurosurgeon and ENT surgeon for the complete clearance of the disease. The histopathological examination was reported as mucormycosis, and the patient was started on inj liposomal amphotericin B. Patient was followed up postoperatively by serial nasal endoscopy and imaging as required.

Conclusion: Mucormycosis in an immunocompetent patient with polyps and dural erosion is a rare entity and hence requires complete removal of the disease process and reconstruction of the dura followed by the inj liposomal Amphotericin B. The patient needs regular clinicradiological follow-up to detect recurrence.

Clinical significance: Awareness of unusual manifestations and complications of paranasal sinus fungal disease is essential.

Keywords: Anterior skullbase, Fungal ball, Mucor, Polyposis.

Clinical Rhinology An International Journal (2019): 10.5005/jp-journals-10013-1351
\end{abstract}

\section{BACKGROUND}

Rhinocerebral mucormycosis, also known as phacomycosis or zygomycosis, is a rare fatal opportunistic infection caused by the family of mucoraceae (Mucor, Rhizomucor, Rhizopus, and Absidia species) that are commonly found in soil and among decaying vegetation. ${ }^{1}$ Several clinical forms have been seen, such as pulmonary, cerebral, gastrointestinal, rhino cerebral mucormycosis, or disseminated form. In patients with malignancy, the lungs are the more likely to be affected, while diabetic patients are more likely to have sino-orbital disease. Cutaneous infection is most likely associated with history of trauma. Rhino-orbital-cerebral is the most common form in diabetic patients. There is not enough data about mucormycosis in immunocompetent patients; some studies showed that cutaneous infection is the most affected organ in apparently healthy patients $(42.5 \%)$ followed by rhino-orbitocerebral mucormycosis (38.2\%). ${ }^{2}$ Management of mucormycosis still represents a big challenge and is based on different strategies that envisage a rapid diagnosis, elimination of risk factors (drugs or underlying diseases), rapid and aggressive antifungal infusion (polyenes) with or without surgical debridment, ${ }^{3,4}$ and lastly with adjuvant therapies in patients refractory or intolerant to polyenebased therapy (posaconazole, deferasirox plus lipid polyenes, recombinant cytokines granulocyte colony-stimulating factor, granulocyte macrophage colony-stimulating factor, or interferon-g, hyperbaric oxygen). ${ }^{5}$

\section{Case Description}

A 36-year-old male office worker presented to outpatient department (OPD) with complaints of recurrent left side nasal discharge and nasal block since 2 years and global dull aching heading ache since 6 months with no history of any comorbidities and vision disturbance or neck stiffness. Nasal endoscopy showed 1,2Department of Otorhinolaryngology, MS Ramaiah Medical College,
Bengaluru, Karnataka, India
${ }^{3}$ Department of Neurosurgery, Vikram Hospital, Bengaluru, Karnataka, India

Corresponding Author: Rajiv $\mathrm{R}$ Sanji, Department of Otorhinolaryngology, MS Ramaiah Medical College, Bengaluru, Karnataka, India, Phone: +919844790345, e-mail: rajivsanji@gmail. com

How to cite this article: Sanji RR, Abey RS, Jagannatha AT. Uncommon Common Cold: Intracranial Fungal Ball. Clin Rhinol An Int J 2019;12(1):24-26.

Source of support: Nil

Conflict of interest: None

multiple pale polyps in the left side nasal cavity, and the right nasal cavity was normal. Computerized tomography (CT) of the paranasal sinuses showed irregularly enhancing mass in the left maxillary and frontal sinus with eroded posterior table of left frontal sinus (Fig. 1). Magnetic resonance imaging (MRI) with contrast was done which showed features suggestive of left side maxillary, ethmoid, and sphenoid sinusitis with leptomeningeal enhancement (Fig. 2).

Hence, a provisional differential diagnosis of invasive fungal sinusitis/fungal ball was made, and the patient underwent surgery under general anesthesia. Surgery involved a team of a neurosurgeon and an ENT surgeon. He was planned for a combined single-sitting transcranial microsurgical and transnasal endoscopic approach to achieve maximum clearance.

\section{Procedure}

A pericranial flap was elevated (Fig. 3), hemicraniectomy was performed, frontal sinus was, opened and multiple fungal balls with

(c) The Author(s). 2019Open Access This article is distributed under the terms of the Creative Commons Attribution 4.0 International License (https://creativecommons. org/licenses/by-nc/4.0/), which permits unrestricted use, distribution, and non-commercial reproduction in any medium, provided you give appropriate credit to the original author(s) and the source, provide a link to the Creative Commons license, and indicate if changes were made. The Creative Commons Public Domain Dedication waiver (http://creativecommons.org/publicdomain/zero/1.0/) applies to the data made available in this article, unless otherwise stated. 


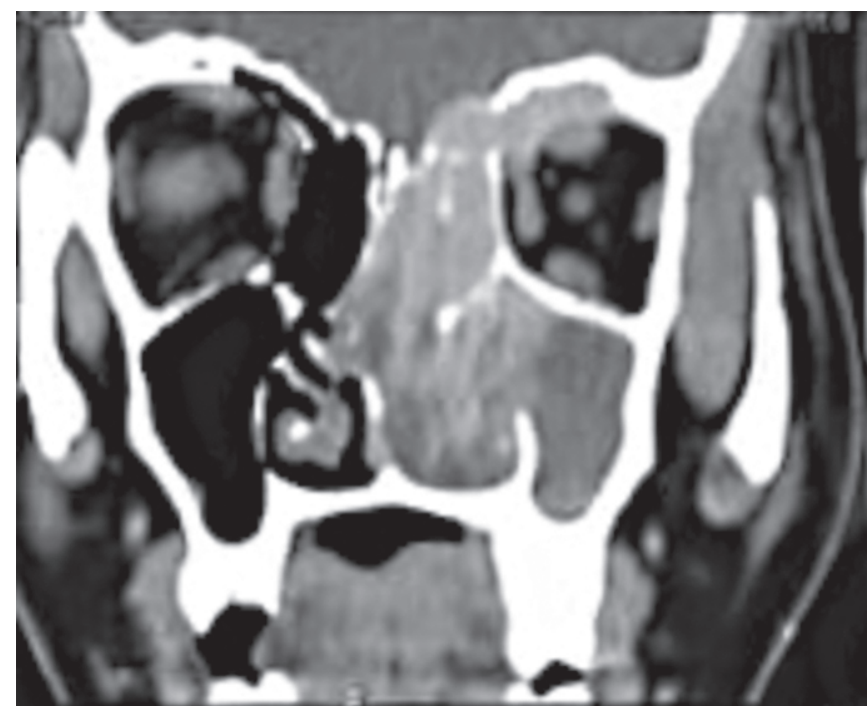

Fig. 1: Coronal computed tomography (CT) slice

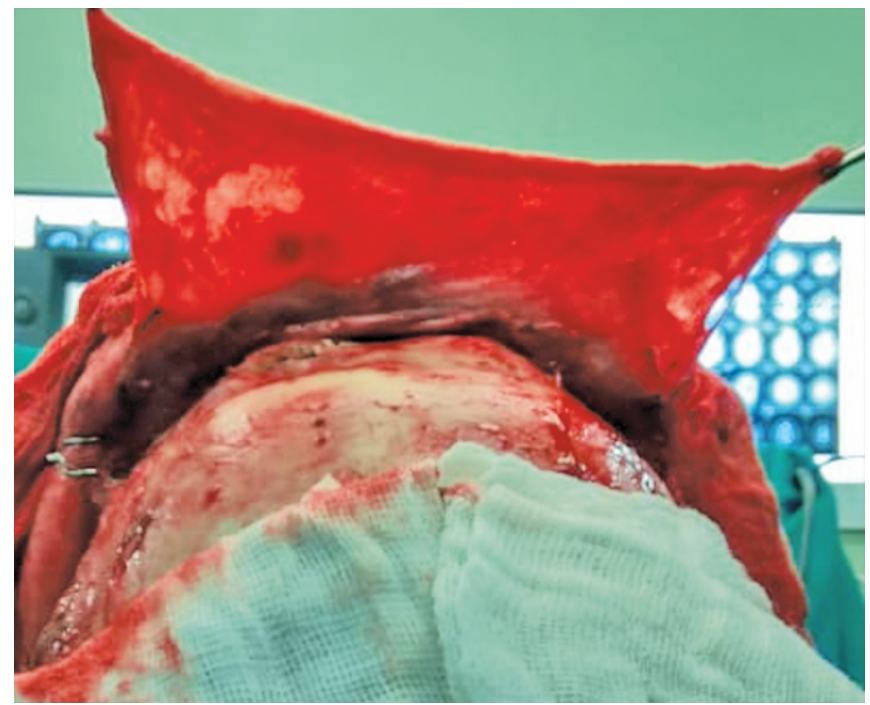

Fig. 3: Pericranial lap

thick tarry mucus was seen in the frontal sinus (Fig. 4), with erosion of the posterior table of frontal sinus and minimal erosion in anterior table. The dura was exposed, erosive changes were seen, and the eroded dura was excised and reconstructed using autologous fat and fibrin glue. The eroded frontal table was debrided and reconstructed using titanium mesh.

Functional endoscopic sinus surgery was performed on the left side, and the maxillary, ethmoid, and sphenoid sinuses were cleared of polyps and fungal debris with fungal balls. The sphenoid sinus was relatively free of fungal balls and could be evacuated with a small spenoidotomy.

Skullbase disease clearance was confirmed transnasally and intracranially by both surgical teams. A wide cribriform and posterior frontal defect was formed after disease clearance.

Reconstruction of the skull base was done using a pericranial flap $^{6}$ and fat from the thigh intracranially and a HaddadBassagasteguy flap ${ }^{7}$ intranasally.

The fungal ball was sent for histopathology suspecting Aspergillosis but was reported as having aseptate hyphae suggestive of mucormycosis. Patient was administered a total dose

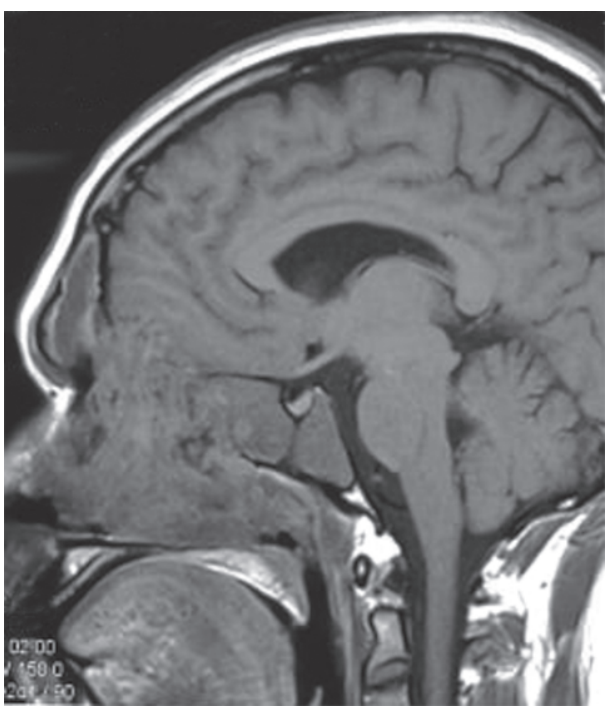

Fig. 2: Sagittal magnetic resonance imaging (MRI)

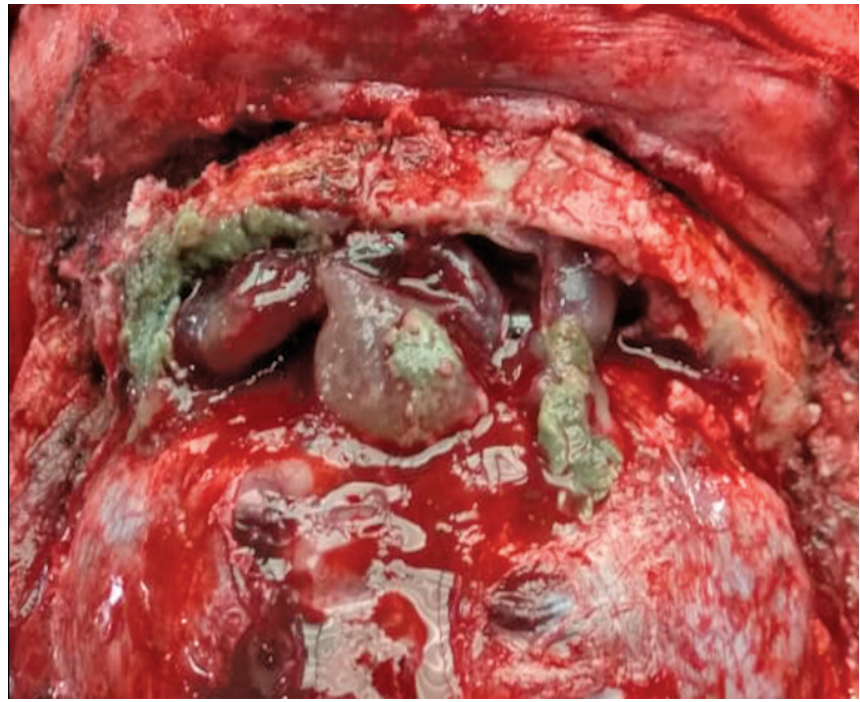

Fig. 4: Frontal sinus muck

of $1.5 \mathrm{~g}$ iv of liposomal Amphotericin B over a period of 2 weeks. He tolerated the treatment well. He was regularly followed weekly for 3 weeks following which a repeat MRI was done which showed absolute clearance of the disease with no signs of recurrence. He was put on prophylactic itraconazole $100 \mathrm{mg}$ orally for 3 weeks following discharge.

Postoperatively nasal endoscopy showed well taken up nasal septal flap with no signs of cerebrospinal fluid leak. There was no postoperative complication like infection or flap give way.

The patient was on OPD follow-up for a period of 1 year following the surgery; after a quiet period, he presented with sinonasal polyposis with ethmoiditis on the right side (non operated side) about 10 months postoperatively with a relatively disease free maxillary and right frontal sinus on CT scan. This responded well to a course of systemic steroid followed by intranasal corticosteroid. He continues to be on outpatient follow-up.

\section{Discussion}

Fungal infections of the paranasal sinuses have been well documented and form a significant portion of the rhinologist's 
workload. While invasive fungal paranasal sinus disease usually presents in immunocompromised individuals with characteristic clinical features of spreading insensate ischemic patches of mucosa followed by necrosis, sinonasal polyps are a well-recognized clinical feature in fungal ball/allergic fungal sinusitis of the paranasal sinuses. ${ }^{8}$ This patient had no features of immunocompromise, and clinical evaluation of this patient was leading to a probable diagnosis of fungal ball with characteristic thick mucus and polyps. The erosion of the posterior table of the frontal sinus was a warning to the clinician that this was not a routine case of fungal ball. Although erosion of the paranasal sinus bones is seen in chronic noninvasive fungal disease of the PNS, ${ }^{9}$ MRI gave an additional surprise with loss of meningeal planes and leptomeningeal enhancement. Preoperative imaging enabled successful coordination of neurosurgeon and rhinologist to operate upon the patient with adequate patient information and preparation. Intraoperative findings again were in favor of fungal ball with characteristic balllike fungal debris and thick muck with the exception of inflamed and friable dura mater over the affected area. Reconstruction of large anterior skullbase defect was successfully accomplished by use of vascular pericranial and nasal septal flaps on either side of the defect coupled with fat obliteration of the defect. Postoperative surprise was the histopathology report of aseptate hyphae leading to a provisional diagnosis of Mucor species infection. Mucor species have also been frequently identified in fungal balls in patients with noninvasive fungal disease of the paranasal sinuses. ${ }^{10,11}$ There are several hypotheses for the erosion of paranasal sinus bone in noninvasive fungal infection, including chronicity of the associated inflammation, bacterial coinfection, and limited local invasion by fungus. ${ }^{5}$ The successful treatment of mucormycosis requires 4 steps: (1) early diagnosis; (2) reversal of underlying predisposing risk factors, if possible; (3) surgical debridement where applicable; and (4) prompt antifungal therapy. ${ }^{3}$ Among patients with rhinoorbital-cerebral disease, CT typically reveals only sinusitis, so CT that indicates the absence of deeper infection does not rule out mucormycosis. MRI is more sensitive than CT for detecting orbital and central nervous system involvement. ${ }^{12}$

While fungal ball does not warrant treatment with Amphotericin $B$, in this patient, we had to initiate amphotericin $B$ treatment due to grave clinical features, such as meningeal involvement and large skullbase erosions.

Use of vascular pedicled flaps on both sides of the defect gave the surgeons confidence in the postoperative result. Fat obliteration of the defect and frontal sinus with cranialization of the frontal sinus eliminated the need for wide transnasal exposure of the frontal sinus and the probable subsequent reconstruction of the posterior table.

Recurrence of nasal polyps on the unoperated side with absence of signs of fungal disease and clinical improvement with medical management indicates a possible pathology of nasal polyposis including a component of allergy to fungal antigens of Mucor species-testing of which is technically challenging.

\section{ConcLusion}

An immunocompetent patient presented with clinical features of sinonasal polyposis with possible fungal ball-unusual extensive skullbase erosions and dura involvement were noted.
Surgical management involved extensive skullbase reconstruction by transcranial and transnasal approaches. Identification of Mucor necessitated postoperative amphotericin-B therapy. Mucor infection may present in fungal balls, invasive fungal sinusitis, and also has a possible role in immune dysfunction in patients with polyps.

\section{Clinical Significance}

Any patient with sinonasal polyposis should be carefully evaluated with appropriate radiological investigations to rule out skullbase complications. In the presence of such complications, a team approach with neurosurgeon and otolaryngologist is important for a clean surgical plan and reconstruction. Fungus identification is important for postoperative decision-making and prognosis.

\section{Ethical and Humane Considerations}

Relavant informed consents were obtained from the patient, the patient's identity has not been disclosed; no clinical trial was conducted.

\section{References}

1. Roden MM, Zaoutis TE, Buchanan WL, et al. Epidemiology and outcome of zygomycosis: a review of 929 reported cases. Clin Infect Dis 2005;41(5):634-653. DOI: 10.1086/432579.

2. Rangel-Guerra RA, Martínez HR, Sáenz C, et al. Rhinocerebral and systemic mucormycosis. clinical experience with 36 cases. J Neurol Sci [Internet] 1996;143(1-2):19-30. DOI: 10.1016/s0022-510x(96)00148-7. Available from: http://www.ncbi.nlm.nih.gov/pubmed/8981294.

3. Selvam M, Pande A, Chakravarthy VM, et al. Invasive rhinocerebral fungal granuloma. Neurol India 2010;58(2):270-276. DOI: 10.4103/0028-3886.63805.

4. Pande A. Fungal infections Prakash Narain Tandon RR, ed. Textbook of Neurosurgery. 3rd ed., Jaypee Publishers; 2012. pp. 815-829.

5. Scheckenbach K, Cornely O, Hoffmann TK, et al. Emerging therapeutic options in fulminant invasive Rhinocerebral mucormycosis. Auris Nasus Larynx 2010;37(3):322-328. DOI: 10.1016/j.anl.2009.09.001.

6. Horowitz JH, Persing JA, Nichter LS, et al. Galeal-pericranial flaps in head and neck reconstruction anatomy and application. Am J Surg [Internet] 1984;148(4):489-497. DOI: 10.1016/0002-9610(84)90375-1.

7. Hadad G, Bassagasteguy L, Carrau RL, et al. A novel reconstructive technique after endoscopic expanded endonasal approaches: vascular pedicle nasoseptal flap. Laryngoscope [Internet] 2006;116(10):1882-1886. DOI: 10.1097/01.mlg.0000234933.37779.e4.

8. Thompson GR, Patterson TF. Fungal disease of the nose and paranasal sinuses. J Allergy Clin Immunol [Internet] 2012;129(2):321-326. DOI: 10.1016/j.jaci.2011.11.039.

9. Jun YJ, Shin JM, Lee JY, et al. Bony changes in a unilateral maxillary sinus fungal ball. J Craniofac Surg [Internet] 2018;29(1):e44-e47. DOI: 10.1097/SCS.0000000000004010. Available from: http://www.ncbi. nlm.nih.gov/pubmed/28953151.

10. Cho HS, Yang HS, Kim KS. Mucormycosis (Mucor fungus ball) of the maxillary sinus. Ear Nose Throat J [Internet] 93(10-11):E18-E22. Available from: http://www.ncbi.nlm.nih.gov/pubmed/25397383.

11. Saydam L, Erpek G, Kizilay A. Calcified Mucor fungus ball of sphenoid sinus: an unusual presentation of sinoorbital mucormycosis. Ann Otol Rhinol Laryngol 2016;106(10):875-877. DOI: 10.1177/000348949710601013.

12. Spellberg B, Walsh TJ, Kontoyiannis DP, et al. Recent advances in the management of mucormycosis: from bench to bedside. Clin Infect Dis 2009;48(12):1743-1751. DOI: 10.1086/599105. 